\title{
Low-dose anthracyclines in childhood Acute Lymphoblastic Leukemia (ALL): no cardiac deterioration more than 20 years post-treatment
}

\author{
C. A. J. Brouwer • J. A. Gietema • M. P. van den Berg • \\ M. T. E. Bink-Boelkens • N. J. Elzenga • J. Haaksma • \\ W. A. Kamps • J. M. Vonk • A. Postma
}

Received: 19 June 2007 / Accepted: 24 August 2007 / Published online: 27 September 2007

(C) Springer Science + Business Media, LLC 2007

\begin{abstract}
Introduction In children with cancer a well-known risk factor for cardiotoxicity is a high cumulative dose of anthracyclines, but little is known about cardiac function in low-dose anthracycline-treated survivors. Also, it is unclear if a safe anthracycline-dose exists at all.

Patients and methods Cardiac function was assessed in 23 long-term ALL-survivors with a median follow-up of 22 years (range 19.5-24.5) post-treatment. Age at diagnosis
\end{abstract}

This study was presented as a poster presentation at the 9th International Conference on Long-term Complications of Treatment of Children and Adolescents for Cancer 2006, Niagara-on-the-Lake, Canada.

C. A. J. Brouwer $(\bowtie) \cdot$ W. A. Kamps · A. Postma

Subdivision Paediatric Oncology, Department of Paediatrics,

University Medical Centre Groningen, University of Groningen, P.O. Box 30 001, 9700 RB Groningen, The Netherlands

e-mail: c.a.j.brouwer@bkk.umcg.nl

\section{J. A. Gietema}

Department of Medical Oncology,

University Medical Centre Groningen, University of Groningen,

Groningen, The Netherlands

M. P. van den Berg · J. Haaksma

Department of Cardiology,

University Medical Centre Groningen, University of Groningen,

Groningen, The Netherlands

M. T. E. Bink-Boelkens • N. J. Elzenga

Subdivision Paediatric Cardiology, Department of Paediatrics, University Medical Centre Groningen, University of Groningen, Groningen, The Netherlands

\section{J. M. Vonk}

Department of Epidemiology,

University Medical Centre Groningen, University of Groningen,

Groningen, The Netherlands and current age were $5.0(2.0-14.0)$ and 29.0 (24.0-39.0) years. All 23 survivors were treated according to DCLSG protocol ALL-5, including 18-25 Gy cranial irradiation. Thirteen of them received $4 \times 25 \mathrm{mg} / \mathrm{m}^{2}$ daunorubicin by randomization. Cardiac evaluation included blood pressure measurement, echocardiography, and (24 h-) electrocardiogram. Results were compared with an earlier assessment at median 12 years post-treatment.

Results None of the survivors had cardiac abnormalities. Cardiac status of daunorubicin-treated survivors showed no deterioration compared with the previous assessment in 1995.

Conclusion and implication for cancer survivors After prolonged follow-up (more than 20 years post-treatment), ALL-survivors treated with low dose daunorubicin had no clinical relevant deterioration of cardiac function.

Keywords ALL - Cardiotoxicity - Daunorubicin . Late effects

\section{Introduction}

During the last few decades, efficacy of childhood cancer treatment improved substantially, which led to an increase in survival of childhood cancer patients. This increased survival is partly due to the introduction of the anthracyclines in the 1970s. Although very effective anti-cancer drugs, anthracyclines may cause cardiac dysfunction [1-3]. The cumulative risk of developing clinical heart failure more than 20 years after anthracycline-treatment has been reported to be 5.5\% [1]. Prevalence of subclinical heart failure was even found in up to $57 \%$ [4]. The risk of developing heart failure after anthracycline-treatment seems to be dose-dependent [5]. At a 
median follow-up of 7.1 years after the first dose of anthracyclines, a recent study showed that survivors treated with less than $150 \mathrm{mg} / \mathrm{m}^{2}$ anthracyclines [i.e. doxorubicin, daunorubicin (DNR), epirubicin and/or idarubicin] had no risk for developing clinical heart failure, while survivors treated with more than $600 \mathrm{mg} / \mathrm{m}^{2}$ had a risk of $14.3 \%$. [1] To determine the possible late development of (sub)clinical cardiac dysfunction in low-dose anthracycline-treated survivors, longitudinal studies with a more prolonged follow-up are warranted.

Earlier, we reported the results of a cross-sectional, nationwide, multi-center cardiac study in 90 long-term survivors of acute lymphoblastic leukemia (ALL) who were randomized to receive low dose anthracyclines in the induction phase (four weekly doses of $25 \mathrm{mg} / \mathrm{m}^{2}$ DNR) [6]. Fifty of these 90 survivors received the low dose of DNR and 40 were treated without DNR. No deterioration of cardiac function and heart rate variability (HRV) was found in the DNR-treated survivors compared with the non-DNRtreated ones. The current study re-assesses cardiac status at a very long follow-up of median 22 years post-treatment in a subset of the earlier studied survivors, namely in those who were treated at a single institution $(n=28)$.

\section{Patients and methods}

\section{Patients}

The initial cohort of survivors treated for childhood ALL at the University Medical Centre Groningen between 1978 and 1984 and participating in a nationwide, multi-center cardiac study in 1995, consisted of 28 patients. All patients were treated according to the DCLSG (Dutch Childhood Leukaemia Study Group) ALL-5 protocol with or without DNR. Furthermore, all patients received a dose of 18 to 25 Gy cranial irradiation as central nervous system prophylaxis. Details about the ALL-5 protocol were described earlier and are summarized in Table 1 [6].

In 1995, 28 institutional ALL-survivors had an assessment of cardiac function at 12 (range, 10.5-15.0) years posttreatment. In 2005, 23 of them were re-assessed 22 (19.5$24.5)$ years post-treatment. Reasons for non-participation in 2005 were second malignancy $(n=1)$ and refusal $(n=4)$. As far as we know, these non-participating survivors had no (clinical) signs of cardiac failure. Characteristics of the 23 participating survivors are summarized in Table 2. Because of randomization during the induction phase of the DCLSG ALL-5 protocol, 13 of the 23 survivors received treatment with four weekly doses of $25 \mathrm{mg} / \mathrm{m}^{2} \mathrm{DNR}$ (DNR+ group), while the others $(n=10)$ received no DNR during the induction phase (DNR- group).
The study protocol was approved by the Ethics Committee of the University Medical Centre Groningen. Written informed consent was obtained from all patients.

\section{Measurements}

Cardiac evaluation consisted of a medical history, full physical examination, blood pressure measurement, Doppler echocardiogram, 12-lead electrocardiogram (ECG) and 24-h ambulatory ECG (24-h ECG).

Standardized measurements of blood pressure were performed in a quiet room in the supine position. Blood pressure was measured twice on the left arm and the lowest blood pressure measurement was taken for analysis. Criteria of hypertension were systolic blood pressure $\geq 140 \mathrm{mmHg}$ and/or diastolic blood pressure $\geq 90 \mathrm{mmHg}$ and/or treatment with antihypertensive drugs. Blood pressure measurements at the current assessment were compared with the results of 1995 .

Echocardiography was performed in order to determine systolic and diastolic function. A single skilled technician performed echocardiography on a General Electric VIVID 7 system with a $2.5 \mathrm{mHz}$ probe. Echocardiography consisted of two-dimensional echocardiogram, color flow mapping and 2D-guided M-mode, blood pool, and tissue Doppler echocardiogram [7]. We measured left ventricular end-diastolic dimension (LVEDD; normal 36-54 mm), left

Table 1 DCLSG ALL5 protocol outline

\begin{tabular}{|c|c|}
\hline Induction treatment & \\
\hline Duration, 6 weeks & Vincristin $6 \times 2 \mathrm{mg} \mathrm{m}^{2}$ week \\
\hline & Prednisone $28 \times 40 \mathrm{mg} \mathrm{m}^{2}$ day \\
\hline & $\begin{array}{l}\text { L-Asparginase } 14 \times 200 \mathrm{E} \mathrm{kg} \\
\text { day in weeks } 4-6\end{array}$ \\
\hline & $\begin{array}{l}\text { Randomization: with or withou } \\
4 \times 25 \mathrm{mg} \mathrm{m}^{2} \text { week } \\
\text { daunorubicin }\end{array}$ \\
\hline Central nervous system $p$ & \\
\hline Duration, $2.5-3$ weeks & Cranial irradiation $18-25$ Gy \\
\hline & $\begin{array}{l}\text { Methotrexate } 12.5 \mathrm{mg} / \mathrm{m}^{2} \\
\text { (maximum } 15 \mathrm{mg} / \mathrm{dose} \text { ) and } \\
\text { prednisone } 12.5 \mathrm{mg} / \mathrm{m}^{2} 5 \times \\
\text { intrathecally }\end{array}$ \\
\hline Maintenance and consolic & \\
\hline Duration, until 2 years & Five weeks 6-mercaptopurine \\
\hline from start of treatment & $50 \mathrm{mg} \mathrm{m}^{2}$ day and \\
\hline & methotrexate $30 \mathrm{mg} \mathrm{m}^{2}$ \\
\hline & week, alternating with \\
\hline & 2 weeks vincristin $2 \mathrm{mg} \mathrm{m}^{2}$ \\
\hline & $\begin{array}{l}\text { week and prednisone } 40 \mathrm{mg} \\
\mathrm{m}^{2} \text { day }\end{array}$ \\
\hline
\end{tabular}

DCLSG Dutch Childhood Leukemia Study Group 
Table 2 Characteristics of the participants

\begin{tabular}{|c|c|c|c|}
\hline & $\begin{array}{l}\text { Total } \\
(n=23)\end{array}$ & $\begin{array}{l}\text { DNR+ } \\
(n=13)\end{array}$ & $\begin{array}{l}\text { DNR- } \\
(n=10)\end{array}$ \\
\hline \multicolumn{4}{|l|}{ Gender } \\
\hline Male/female & $9 / 14$ & $6 / 7$ & $3 / 7$ \\
\hline \multicolumn{4}{|c|}{ Age (years) at diagnosis } \\
\hline $\begin{array}{l}\text { Median } \\
\text { (range) }\end{array}$ & $5.0(2.0-14.0)$ & $5.0(2.0-14.0)$ & $5.0(2.0-12.0)$ \\
\hline \multicolumn{4}{|c|}{ Age (years) at follow-up } \\
\hline $\begin{array}{l}\text { Median } \\
\text { (range) }\end{array}$ & $\begin{array}{l}29.0 \\
(24.0-39.0)\end{array}$ & $\begin{array}{l}30.0 \\
(24.0-39.0)\end{array}$ & $\begin{array}{l}27.5 \\
(26.0-34.0)\end{array}$ \\
\hline \multicolumn{4}{|c|}{ Follow-up period (years) } \\
\hline $\begin{array}{l}\text { Median } \\
\text { (range) }\end{array}$ & $\begin{array}{l}21.5 \\
(19.5-24.5)\end{array}$ & $\begin{array}{l}22.0 \\
(20.0-24.5)\end{array}$ & $\begin{array}{l}20.8 \\
(19.5-23.5)\end{array}$ \\
\hline
\end{tabular}

No significant differences in sex, age at diagnosis, age at follow-up, follow-up period and dose of cranial irradiation between daunorubicintreated survivors and non-daunorubicin-treated survivors.

$D N R+$ Treated with $4 \times 25 \mathrm{mg} / \mathrm{m}^{2}$ daunorubicin, $D N R-$ treated without daunorubicin

ventricular end-systolic dimension (LVESD; normal 23$40 \mathrm{~mm}$ ), intraventricular septum end-diastolic (IVSed; normal 7-11 mm), and end-diastolic left ventricular posterior wall thickness (LVPWed; normal 7-11 mm) in the M-mode, obtained in the standard left ventricular parasternal long axis. Shortening fraction (SF) and wall motion score index (WMSI) were indicators of systolic function. SF was calculated with the formula: (LVEDD-LVESD)/LVEDD $\times$ $100 \%$. WMSI was calculated by visually scoring between 1 and 4 of each of the 16 segments of the left ventricle $(1=$ normokinesia; 2 =hypokinesia; 3 =akinesia; 4 =dyskinesia). The scores of each segment were added and the total score was divided by the number of analysed segments. A SF above $29 \%$ and a WMSI of 1.00 are considered to be normal. Diastolic function measurements included mitral valve inflow velocities [early (E) as well as late (A) in diastole] and diastolic tissue velocity at the mitral valve annulus [tissue velocity imaging of early diastole (TVI Et); parameter of diastolic function independent of left ventricular filling]. E/A ratio $<1.00$ was considered abnormal and may represent diastolic dysfunction. A mean TVI Et $<8.0 \mathrm{~cm} / \mathrm{s}$ was considered diastolic dysfunction [8].

A standard 12-lead ECG was recorded and analysed by a single observer for flattened T-waves, pathological Q-waves or a prolonged QTc. T-waves were characterised as flattened if three or more precordial leads and three or more standard leads had an amplitude less than +2 or $-2 \mathrm{~mm}$. In males QTc $>0.44 \mathrm{~s}$ was considered abnormal and in females QTc $>0.46 \mathrm{~s}$.

The 24-h ECG was analysed on a GE Marquette Holter system by an experienced Holteranalyst. All Holters were analysed for rhythm and conduction disturbances. Ventricular arrhythmias were classified according to the Lown's criteria [9]. Lown 4 or higher was considered abnormal.

For analysis of HRV, data were transformed to a $\mathrm{PC}$ and custom made software (COHWIN) was used to analyse HRV [10]. Both time domain and frequency domain parameters were calculated in accordance with the recommendations for analysis of HRV [11]. For frequency domain parameters 5 min segments were used. Segments containing more than $10 \%$ ectopics as well as nonstationary segments were excluded from the analysis. We determined the spectral power over three frequency regions of interest: LF, low frequency power $(0.04-0.15 \mathrm{~Hz})$; HF, high frequency power $(0.15-0.4 \mathrm{~Hz})$; and $\mathrm{TP}$, total power (0.01-1.00 Hz). In particular the parameters rMSSD (root mean square of successive difference) and HF reflect parasympathetic activity. HRV is heart rate-, and therefore, gender- and age-dependent [10]. In 1995 no abnormalities in HRV were found [6]. Therefore, we only compared HRV measurements of 2005 with sex- and age-matched controls $(n=23)$, recruited from a group of 419 healthy subjects, who were investigated previously [10].

\section{Statistical analyses}

Statistical analyses were performed in SPSS Inc. version 12 by the non-parametric Mann-Whitney, Wilcoxon's, Chi squared and McNemar tests. Data were reported as median (range). Two-sided $p$ values $\leq 0.05$ were considered significant.

\section{Results}

Clinical parameters and physical examination

No significant differences in sex, age at diagnosis, age at follow-up and follow-up period were found between DNR+ survivors and DNR- survivors (Table 2). Furthermore, there was no difference in dose of cranial irradiation between both groups. DNR+ survivors as well as DNRsurvivors received median 25 (range, 18-25) Gy cranial irradiation. Three of the 13 DNR+ ALL-survivors complained of palpitations and one of dyspnoea with exertion, while none of the DNR- ALL-survivors had cardiac symptoms.

At the current assessment, the entire group of ALLsurvivors did not have a higher prevalence of hypertension compared with 1995 ( $p=1.00)$. Furthermore, no differences in prevalence of hypertension were found between DNR+ and DNR - survivors $(p=0.56)$ and DNR + survivors as well as DNR- survivors had no higher prevalence of hypertension in 2005 compared with 1995 ( $p=1.00$; Table 3). 
Table 3 Results of physical examination and echocardiography

\begin{tabular}{|c|c|c|c|c|c|c|}
\hline & \multicolumn{3}{|c|}{ ALL survivors in $1995(n=23)$} & \multicolumn{3}{|c|}{ ALL survivors in $2005(n=23)$} \\
\hline & $\begin{array}{l}\text { All survivors } \\
(n=23)\end{array}$ & $\begin{array}{l}\mathrm{DNR}+ \\
(n=13)\end{array}$ & $\begin{array}{l}\text { DNR- } \\
(n=10)\end{array}$ & $\begin{array}{l}\text { All survivors } \\
(n=23)\end{array}$ & $\begin{array}{l}\mathrm{DNR}+ \\
(n=13)\end{array}$ & $\begin{array}{l}\text { DNR- } \\
(n=10)\end{array}$ \\
\hline $\begin{array}{l}\text { Hypertension (blood pressure } \\
\geq 140 / 90 \mathrm{mmHg} \text { ) }\end{array}$ & $2 / 23(9 \%)$ & $1 / 13(8 \%)$ & $1 / 10(10 \%)$ & $3 / 23(13 \%)$ & $1 / 13(8 \%)$ & $2 / 10(20 \%)$ \\
\hline $\mathrm{SF}(\%)^{\mathrm{a}}$ & $34(31-39)$ & $33(31-39)$ & $35(32-38)$ & $36^{\mathrm{b}}(29-52)$ & $36(29-52)$ & $37(33-44)$ \\
\hline $\mathrm{E} / \mathrm{A}$ ratio $^{\mathrm{a}}$ & $1.8(1.1-2.9)$ & $1.9(1.4-2.9)$ & $\begin{array}{l}1.8 \\
(1.1-2.4)\end{array}$ & $1.7(0.8-2.4)$ & $\begin{array}{l}1.7^{\mathrm{c}} \\
(1.3-2.4)\end{array}$ & $\begin{array}{l}1.7 \\
(0.8-2.4)\end{array}$ \\
\hline IVSed $(\mathrm{mm})^{\mathrm{a}}$ & $9.0(8-12)$ & $9.0(8-11)$ & $9.0(8-12)$ & $8.0^{\mathrm{d}}(6-11)$ & $8.0^{\mathrm{e}}(6-9)$ & $8.5(7-11)$ \\
\hline LVPWed $(\mathrm{mm})^{\mathrm{a}}$ & $9.0(7-10)$ & $9.0(7-10)$ & $9.0(7-10)$ & $8.0(5-10)$ & $8.0(5-10)$ & $8.0(6-10)$ \\
\hline $\operatorname{LVESD}(\mathrm{mm})^{\mathrm{a}}$ & $30.0(25-36)$ & $30.0(25-36)$ & $\begin{array}{l}30.5 \\
(26-33)\end{array}$ & $29.0^{\mathrm{f}}(22-34)$ & $29.0(22-34)$ & $\begin{array}{l}29.0 \\
(22-33)\end{array}$ \\
\hline $\operatorname{LVEDD}(\mathrm{mm})^{\mathrm{a}}$ & $46.0(41-53)$ & $46.0(41-53)$ & $\begin{array}{l}46.0 \\
(41-51)\end{array}$ & $46.0(39-51)$ & $47.0(40-51)$ & $\begin{array}{l}45.5 \\
(39-49)\end{array}$ \\
\hline
\end{tabular}

$A L L$ Acute lymphoblastic leukemia, $D N R+$ treated with $4 \times 25 \mathrm{mg} / \mathrm{m}^{2}$ daunorubicin $(D N R), D N R-$ treated without DNR, $S F$ shortening fraction, IVSed intraventricular septum end-diastolic, LVPWed end-diastolic left ventricular posterior wall thickness, LVESD left ventricle systolic diameter, $L V E D D$ left ventricle end-diastolic diameter

${ }^{\mathrm{a}}$ Median (range)

${ }^{\mathrm{b}}$ Comparison of SF between 1995 and 2005 of all survivors, $p=0.03$

${ }^{\mathrm{c}}$ Comparison of E/A ratio between 1995 and 2005 in survivors treated with DNR, $p=0.02$

${ }^{\mathrm{d}}$ Comparison of IVSed between 1995 and 2005 in all survivors, $p=0.001$

${ }^{\mathrm{e}}$ Comparison of IVSed between 1995 and 2005 in survivors treated with DNR, $p=0.004$

${ }^{\mathrm{f}}$ Comparison of LVESD between 1995 and 2005 in all survivors, $p=0.02$

\section{Echocardiography}

The results of echocardiography are summarized in Table 3. All ALL-survivors, DNR+ as well as DNR-, showed normal systolic function (all survivors had a SF $>29 \%$ and a WMSI of 1.00). Median SF was higher in 2005 compared with $1995(p=0.03)$. Only one of the ALL-survivors, treated without DNR, showed diastolic dysfunction. This was a 31-year-old woman with an E/A ratio of 0.84 and a mean TVI Et of $6.0 \mathrm{~cm} / \mathrm{s}$ and with a mildly elevated blood pressure (137/92). For the whole group of ALL-survivors diastolic function (measured as E/A ratio) tended to decrease compared with $1995(p=0.06)$. Furthermore, compared with 1995, we found a decreased IVSed ( $p=$ $0.001)$, a decreased LVESD $(p=0.02)$ and LVPWed tended to be thinner $(p=0.06)$. However, at the assessment of 2005, all survivors had a IVSed, LVPWed, LVEDD and LVESD within normal range, except for one DNR+ survivor with a decreased IVSed $(6 \mathrm{~mm})$ and LVPWed $(5 \mathrm{~mm})$ and for one DNR- survivor with a decreased LVPWed (6 mm). At the assessments of 1995 and 2005, no differences were found in cardiac parameters between DNR+ and DNR- ALL-survivors.

In the DNR+ survivors, E/A-ratio decreased significantly compared with $1995(p=0.02)$, although all values were still within normal limits. The decline in E/A ratio in the DNR+ survivors was not significantly different from the decline in the DNR- group $(p=0.11)$. In addition, mean
TVI Et (only measured in the cardiac assessment of 2005) showed no difference in TVI Et between DNR+ and DNRsurvivors $[10.9(9.0-12.9)$ vs $10.2(6.0-12.6) \mathrm{cm} / \mathrm{s}, p=$ 0.42]. In the DNR+ survivors, the IVSed decreased compared with $1995(p=0.004)$. The decline in IVSed in the DNR+ survivors was not significantly different from the decline in the DNR- group $(p=0.65)$. Furthermore, LVPWed and LVESD tended to decrease, however, not significantly ( $p=0.08$, respectively, $p=0.09$ ). No significant differences were found in SF and LVEDD $(p \geq 0.10$; Table 3). In the DNR- survivors, IVSed tended to decrease $(p=0.06)$, while SF, E/A-ratio, LVPWed, LVESD and LVEDD showed no significant differences between 2005 and $1995(p \geq 0.10)$.

\section{(24-h) ECG}

In 2005, 12-lead ECG showed flattened T-waves in 7/23 $(30 \%)$ survivors. DNR+ survivors had no more often abnormal flattened T-waves compared with DNR- survivors $(p=1.00)$. None of the survivors had pathological Q-waves or a prolonged QTc.

All survivors underwent 24-h ECG. Median (range) heart rate of the survivors was $76(67-88) / \mathrm{min}$. All survivors had sinus rhythm on their ECGs with normal atrioventricular conduction. One DNR+ survivor had sporadic (less than 100/24 h) premature ventricular contractions (Lown 1) and two DNR- survivors had ventricular couplets (Lown 4). 
None of the survivors showed sustained (supra) ventricular tachycardia. At the earlier assessment in 1995, two survivors had Lown 1, one Lown 2 and one Lown 4.

HRV was evaluable in all survivors and the results were compared with age- and sex-matched controls $(n=$ 23). No abnormalities in HRV were found. All time and frequency domain parameters were comparable with those of the controls, except rMSSD (time domain parameter). The rMSSD was higher in patients compared with controls [median (range) $54.0 \mathrm{~ms}(26.8-217.4)$ vs $37.7 \mathrm{~ms}$ (16.2$102.3) ; p=0.02]$. Furthermore, no significant differences in time and frequency domain parameters were found between DNR+ and DNR - survivors. Also at the cardiac assessment of 1995, no abnormalities in HRV were found.

\section{Discussion}

In this longitudinal cardiac study with an extended followup, we found no deterioration of cardiac function and no signs of autonomic dysfunction in ALL-survivors irrespective of their treatment with DNR.

Until now, longitudinal cardiac studies in childhood cancer survivors are scarce, especially those with an extended follow-up period. Recently, we published the results of a cardiac follow-up study in bone tumor survivors and found progressive cardiac dysfunction more than 20 years post-treatment [12]. All these survivors had been treated with medium to high dose of anthracyclines. Even less is known about the very long-term follow-up in survivors treated with low-dose of anthracyclines. Lipshultz et al. found that, at median 12 years post-treatment, SF was relatively normal in the low-dose group $[n=25$; less than $300 \mathrm{mg} / \mathrm{m}^{2}$ doxorubicin; mean $z$ score of SF $-0.59(-1.78$ to 0.61)]. However, they stated that even patients who received a cumulative dose as low as $45 \mathrm{mg} / \mathrm{m}^{2}$ doxorubicin $(n=18)$, eventually experienced cardiac abnormalities, such as significantly reduced left ventricular mass and dimension [2]. A dose-related effect of subclinical cardiotoxicity was found in other studies [5, 13]. A recent cross-sectional study [with a median follow-up of 7.1 years (range 0.0128.4)] demonstrated no clinical heart failure in survivors who had been treated with less than $150 \mathrm{mg} / \mathrm{m}^{2}$ anthracyclines [1]. In this study, data were collected from medical records and questionnaires (filled out by the general practitioners of the survivors). The results of the present study support the finding that low-dose DNR might be safe, as we found neither clinical nor subclinical cardiac abnormalities after a much longer follow-up of 22 years post-treatment.

Compared with the first cardiac assessment in 1995, we found a decrease in E/A-ratio in the DNR+ survivors $(n=$ 13), although all measurements were within normal limits. As the recent cardiac assessment of 2005 was about
10 years after the first one, the decrease in E/A ratio can be considered age-related. This is in accordance with Spirito et al. [14] who described the age-related decline of $\mathrm{E} / \mathrm{A}$ ratio. In addition, no significant difference in the decline in E/A ratio was found between DNR+ survivors and DNR- survivors and mean TVI Et, measured in 2005, was not different between DNR+ and DNR- survivors. This makes a cardiotoxic effect of DNR unlikely.

At the 2005 assessment, DNR+ survivors showed a decrease of IVSed compared with $1995(p=0.004)$ and LVPWed showed a tendency to decrease compared with 1995 ( $p=0.08$ ), although most values as such were still within the normal range. Lipshultz et al. [2] found a statistically significant change in LVPWed over time and found no dose-related differences. Furthermore, Sörensen et al. [5] found a significantly decreased wall thickness in Wilms' tumour survivors at mean 11.1 years since treatment, however these survivors received a much higher dose of anthracyclines (mean, $301 \mathrm{mg} / \mathrm{m}^{2}$ ) and the decreased wall thickness was found in combination with a decreased SF. On the other hand, in the same study cardiac function was also studied in ALL-survivors treated with a lower dose of anthracyclines (mean, $180 \mathrm{mg} / \mathrm{m}^{2}$ ) and in this group they found no decrease in wall thickness and SF at mean 10.3 years since treatment. In addition, others showed no statistically difference in LVPWed between patients treated with median $240 \mathrm{mg} / \mathrm{m}^{2}$ anthracyclines and healthy controls, however their median follow-up was only 6.3 years, while our median follow-up was 22 years [3]. We are not aware of studies that noticed a decreased IVSed in childhood cancer survivors treated with anthracyclines. It can be hypothesized that a decreased IVSed and LVPWed might be considered as precursors of future cardiac dysfunction. However, the finding of a normal HRV in the low-dose DNR+ survivors makes this less likely. HRV, reflecting autonomic function, has been described as a sensitive marker of (early) subclinical cardiotoxicity [15]. A previous study in long-term cancer survivors showed progressive deterioration of HRV in survivors treated with medium to high dosages of anthracyclines more than 20 years post-treatment, together with systolic and diastolic dysfunction [12]. Normal HRV results might underline the finding that no cardiac damage has occurred. In order to gain insight in the prognostic significance of echocardiography parameters (such as IVSed and LVPWed) and HRV, continuous follow-up is warranted in these survivors.

\section{Limitations}

The echocardiography parameters we used in our study were not quite the same as those used by other authors. Mostly, the parameters of systolic and diastolic left 
ventricle function used in the several cardiac studies performed in childhood cancer survivors are readily comparable. In paediatric cardiology, SF is commonly used as a primary indicator of systolic function $[2,3,5,13]$. For a reliable comparison, we also used SF to indicate systolic function. Additionally, WMSI was used as a more sophisticated method to assess systolic function. A difference between our study and those of Lipshultz et al. [2] and Nysom et al. [13] is the use of $z$ scores to describe the cardiac parameters. Their studies (mainly) concerned children. $Z$ scores are commonly used in paediatric cardiology to adjust for changes in growth, but are less commonly used in adult cardiology. In our current study we did not use $z$ scores as the age of all participants was $\geq 18$ years. Diastolic function in anthracycline-treated childhood cancer survivors was studied by Elbl et al. [3], who used a combination of parameters to indicate diastolic function, e.g. E/A ratio, deceleration time, and isovolemetric relaxation time. We used a slightly different combination of parameters to indicate diastolic function, e.g. E/A ratio and TVI Et. TVI Et is an interesting new parameter for assessment of diastolic function and it is more sensitive as it is independent of left ventricular filling. The use of not exactly the same methods might impede comparison of our results with those of others. However, the use of new, more sophisticated and sensitive parameters in assessment of systolic and diastolic function (e.g. WMSI and TVI Et) decreases the chance of underestimation of cardiac dysfunction in low-dose anthracycline-treated childhood cancer survivors. Another limitation of our study is the small cohort of survivors. This causes less power in the statistical analysis. On the other hand, the value of our study is its very long-term follow-up with two subsequent echocardiography evaluations in survivors treated with low dose of anthracyclines.

In conclusion, no clinical relevant echocardiographic abnormalities were found in childhood ALL-survivors treated with a cumulative dose of $100 \mathrm{mg} / \mathrm{m}^{2}$ DNR. The prognostic significance of the changes in some cardiac parameters, like IVSed, LVPWed and E/A ratio, is still largely unknown and has to be elaborated. Therefore, we need to continue sequential follow-up assessments, also in survivors treated with lower doses of anthracyclines.

Acknowledgment This study was performed with financial support of the foundation "The Quality of Life Gala". The authors are indebted to Dr. L. A. J. Rammeloo for his help in preparing the data of this manuscript.

Conflict of interest statement None declared.

\section{References}

1. van Dalen EC, van der Pal HJ, Kok WE, Caron HN, Kremer LC. Clinical heart failure in a cohort of children treated with anthracyclines: a long-term follow-up study. Eur J Cancer. 2006;42:3191-8.

2. Lipshultz SE, Lipsitz SR, Sallan SE, Dalton VM, Mone SM, Gelber RD, et al. Chronic progressive cardiac dysfunction years after doxorubicin therapy for childhood acute lymphoblastic leukemia. J Clin Oncol. 2005;23:2629-36.

3. Elbl L, Hrstkova H, Chaloupka V. The late consequences of anthracycline treatment on left ventricular function after treatment for childhood cancer. Eur J Pediatr. 2003;162:690-6.

4. Kremer LC, van der Pal HJ, Offringa M, van Dalen EC, Voute PA. Frequency and risk factors of subclinical cardiotoxicity after anthracycline therapy in children: a systematic review. Ann Oncol. 2002;13:819-29.

5. Sorensen K, Levitt GA, Bull C, Dorup I, Sullivan ID. Late anthracycline cardiotoxicity after childhood cancer: a prospective longitudinal study. Cancer. 2003;97:1991-8.

6. Rammeloo LA, Postma A, Sobotka-Plojhar MA, Bink-Boelkens MT, Berg A, Veerman AJ, et al. Low-dose daunorubicin in induction treatment of childhood acute lymphoblastic leukemia: no long-term cardiac damage in a randomized study of the Dutch Childhood Leukemia Study Group. Med Pediatr Oncol. 2000;35:13-9.

7. Cheitlin MD, Armstrong WF, Aurigemma GP, Beller GA, Bierman FZ, Davis JL, et al. ACC/AHA/ASE 2003 Guideline Update for the Clinical Application of Echocardiography: summary article. A report of the American College of Cardiology/American Heart Association Task Force on Practice Guidelines (ACC/AHA/ ASE Committee to Update the 1997 Guidelines for the Clinical Application of Echocardiography). J Am Soc Echocardiogr. 2003;16:1091-110.

8. Schiller NB, Shah PM, Crawford M, DeMaria A, Devereux R, Feigenbaum H, et al. Recommendations for quantitation of the left ventricle by two-dimensional echocardiography. American Society of Echocardiography Committee on Standards, Subcommittee on Quantitation of Two-Dimensional Echocardiograms. J Am Soc Echocardiogr. 1989;2:358-67.

9. Lown B, Wolf M. Approaches to sudden death from coronary heart disease. Circulation. 1971;44:130-42.

10. Haaksma J, Brouwer J, Mulder LJM, et al. Heart rate dependent changes in spectral analysis. Computers in cardiology. Los Angeles, CA: Computer Society Press; 45-48, 1994.

11. Heart Rate Variability. Standards of measurement, physiological interpretation and clinical use. Task force of the European Society of Cardiology and the North American Society of Pacing and Electrophysiology. Circulation. 1996;93:1043-65.

12. Brouwer C, Gietema J, van den Berg M, Bink-Boelkens M, Elzenga $\mathrm{N}$, Haaksma $\mathrm{J}$, et al. Long-term cardiac follow-up in survivors of a malignant bone tumour. Ann Oncol. 2006;17:1586-91.

13. Nysom K, Holm K, Lipsitz SR, Mone SM, Colan SD, Orav EJ, et al. Relationship between cumulative anthracycline dose and late cardiotoxicity in childhood acute lymphoblastic leukemia. J Clin Oncol. 1998; 16:545-50.

14. Spirito P, Maron BJ. Influence of aging on Doppler echocardiographic indices of left ventricular diastolic function. Br Heart J. 1988;59:672-9.

15. Tjeerdsma G, Meinardi MT, Der Graaf WT, van den Berg MP, Mulder NH, Crijns HJ, et al. Early detection of anthracycline induced cardiotoxicity in asymptomatic patients with normal left ventricular systolic function: autonomic versus echocardiographic variables. Heart. 1999;81:419-23. 